\title{
Executing IoT Processes in BPMN 2.0: Current Support and Remaining Challenges
}

\author{
Faruk Hasić and Estefanía Serral Asensio \\ Department of Information Management, Modelling and Simulation \\ KU Leuven, Warmoesberg 26, 1000 Brussels, Belgium \\ faruk.hasic@kuleuven.be; estefania.serralasensio@kuleuven.be
}

\begin{abstract}
The Internet of Things (IoT) has seen a vast surge in recent years, both in academia and in industry. Business processes are starting to take advantage of IoT to interact with the increasingly connected and dynamic physical environment. Recent literature has focused on analysing how this interaction between processes and IoT devices can be modelled, i.e. how the process can be described for discussion purposes. However, little attention is given to configuring the execution of these IoT-based processes. IoT-enhanced processes should indeed be able to incorporate IoT devices in the endeavour of process execution. This paper discusses research challenges in IoT process configuration and execution using the executable Business Process Model and Notation 2.0 (BPMN 2.0) standard. As such, we constitute an agenda for future research.
\end{abstract}

Keywords. Internet-of-Things, IoT, Internet-of-Things Processes, IoT Processes, Process Execution, IoT Process Execution, Process Automation, IoT Process Automation.

\section{INTRODUCTION}

The network of interconnected devices that constitute the IoT [1] keeps growing. A plethora of devices are used to facilitate both businesses and individuals in their daily endeavours. The application areas are both vast and diverse, ranging from smart home environments [2] to medical monitoring systems [3]. Usually, a single device does not suffice to encapsulate the physical complexity and therefore multiple devices are combined to render a system that accommodates the reality. Business processes can benefit from IoT by interacting with these devices to steer their behaviour. Ideally, the business process is in turn capable of adapting to its execution environment by tailoring the process to case-specific needs as demanded by the context. This integration of IoT and business processes has enjoyed significant attention in literature [4], and in particular when it comes to the integrated modelling of processes and IoT devices [5]. This interaction between IoT devices and the business process remains of paramount importance during the execution of the process as well. However, very few researchers have investigated this topic, while only providing partial solutions. In this paper, we approach that gap by contributing an incomplete yet indispensable set of research challenges that need to be tackled to achieve IoT process execution using the Business Process Model and Notation 2.0 (BPMN 2.0) standard [6]. We focus on BPMN 2.0 since it is an executable and widely accepted standard in industry and academia. Furthermore, BPMN 2.0 adheres to the modeldriven development (MDD) paradigm, which recognises that models rather than computer programs are the primary focus and drive of software development. As such, models need to be detailed enough to be executable. Though detailed, models should be abstract of the technology used, as the technology should be made explicit during the execution of the system.

This paper is structured as follows. Section III provides background information on process execution, the Internet of Things, the link between IoT and business processes, and the related work on the execution of IoT processes. Section III provides a real-life IoT healthcare running example which will be used to illustrate the challenges. Section IV discusses which elements of BPMN 2.0 are suitable for IoT process execution. In Section $\mathrm{V}$ we identify a set of challenges that need to be tackled to guarantee the correct execution of IoT-enhanced processes, and we identify works that partially address these challenges. Finally, Section VI concludes and provides directions for future research.

\section{BACKGROUND AND RELATED WORK}

In this section, we give an overview of concepts and research areas that are relevant for the execution of IoTenabled business processes.

\section{A. The IoT paradigm}

IoT refers to the ever-growing network of interconnected devices. Generally speaking, an IoT environment is populated by two kinds of devices: sensors and actuators. Sensors serve as a mechanism to capture reality and are therefore the source of IoT data towards the system. Actuators on the other hand operate in the reverse direction, i.e. they change the reality by activating a physical reaction that is initiated by the system. Typical sensors are for example humidity sensors, light sensors, camera sensors, or heart rhythm sensors. On the other hand, typical actuators can be an electric motor, a heating actuator, a sprinkler system, or a speaker. Data collected by IoT devices is considered to be part of the context information [7], [8], i.e. the information describing the conditions under which the system operates. Relevant context information might for instance include the location of an object, the humidity level, and the temperature. Thus, IoT devices aid in digitising the context that manifests itself in the physical environment.

\section{B. Business processes and their execution}

The field of Business Process Management (BPM) is concerned with defining and executing business activities within 
an organisation. The more context-intensive the environment in which the processes takes place, the more challenges arise regarding the modelling and execution of said processes. A process can be specified and executed using the BPMN 2.0 standard. The models expressed in BPMN 2.0 and saved in XMI [9], are interpreted by a process engine, like Camunda [10], to execute the corresponding tasks as specified in the models.

\section{IoT business processes}

Recent literature has focused on incorporating IoT into BPM [4], [11]. IoT and business process integration can be twofold. By definition, IoT business processes are enacted in a dynamic and highly connected physical environment. Since IoT is a technology that can be used to digitalise the context of a system, the process is granted the capability of both understanding its context and of changing it through IoT devices. This was also firmly stressed in [4], indicating that IoT technology can help concretising abstract process models and help the processes deal with new situations. Therefore, on one side IoT devices can be used to digitalise the context of a business process and consequently to incorporate the context into the process execution. That way, an abstract process model can be made concrete for context-aware execution [4]. On another side, IoT devices can be used to automate tasks. This can be a physical task, such as opening the curtains in a smart home, or a digital task, such as sending a message or notification to a system or a human user.

Most papers on the cross section of IoT and process management mainly deal with the integrated modelling of IoT devices in business process models. Examples can be found in [12]. Few works, however, do touch on the subject of configuring IoT processes for execution, e.g. [2]. Most of them rely on code generation, such as [13]. Others use existing process engines for model interpretation, while resorting to code generation for the concepts not supported by the process engine [14]. Another approach is to avoid code generation and to extend an existing process engine to support the newly introduced constructs [15], or to develop an entirely new custom process engine [16].

\section{RunNing ExAmple of AN IOT PRocess}

Consider a patient health monitoring system for a person diagnosed with the Chronic Obstructive Pulmonary Disease (COPD). COPD is a disease that obstructs the lungs and obstructs the airflow and breathing of the patient. Acute attacks of the disease can happen. In that case the patient can experience uncomfortable complications such as fast breathing, a fast heart rate, hyperactive use of muscles, and a cold skin [3]. In scientific literature it has been recognised as well that an IoT-based patient monitoring process can help increase the life quality of the patient and decrease the risks that are inherent to the disease [3]. Multiple sensors and wearable technologies exist that can collect patient data relevant for the patient monitoring process [3]:

- Gait sensors check the posture and walking of the patient.
- Electrocardiogram (ECG) sensors monitor the heart.

- Respiratory sensors check the breathing rate.

- Skin temperature sensors monitor the skin temperature.

- Muscular Electromyography (EMG) sensors monitor the muscle activity.

- Blood pressure sensors measure the blood pressure.

All these sensors collect measurements on the patient's health. IoT settings are often data- and decision-intensive. Note that a single sensor or even a few sensors combined are not enough to capture the COPD. For instance, the patient might take a walk outside in the winter and a sensor registers a low skin temperature. In that case, the patient is not necessarily suffering from COPD at that moment. However, an expert can build patient-specific decision rules to capture COPD in such a monitoring system. For instance, if the sensors register a low skin temperature, a short and fast breathing rate, together with high blood pressure, the monitoring process might decide that the patient is suffering an attack and running out of oxygen. In such a situation the process can trigger the administration of an oxygen mask to the patient. An illustrative COPD monitoring IoT process is given in Figure 1. First the heart rhythm of the patient is checked and the emergency alarm is activated if needed. Afterwards, the severeness of the COPD is assessed, and given the assessment, the process ends or treatments with oxygen masks or inhalers are carried out.

\section{BPMN 2.0 SUPPORT FOR IOT PROCESS EXECUTION}

To achieve IoT process execution, IoT devices must be linked to process elements as described in Section II-C. The BPMN 2.0 standard offers different mechanisms that could be used to establish this link. We can group these mechanisms as follows:

1) Tasks. The latest BPMN standard defines three types of automated tasks:

a) Script tasks have scripts attached to them that are written in a language that the process engine can parse and consequently execute in an automatic fashion.

b) Service tasks are tasks that invoke an application or service to automatically complete the task. Since IoT devices can be managed by IoT services that are invocable by other systems, the service tasks are a suitable mechanism to link the IoT infrastructure to the executable business process. The service task can forward a method call to, for instance, a Java delegate class, which in turn implements an IoT service. That way, communication and data propagation between IoT devices and the business process can be implemented.

c) Business rule tasks can be used to incorporate IoT resources into the business process. IoT data collected by the IoT devices can have the role of input data to the decision logic hidden behind a business rule task.

Figure 2 depicts the task types that are suitable for modelling IoT processes, i.e. a script, service, and business rule task according to the BPMN 2.0 standard [6].

2) Events. Another way to establish communication between IoT devices and the business process is the use 


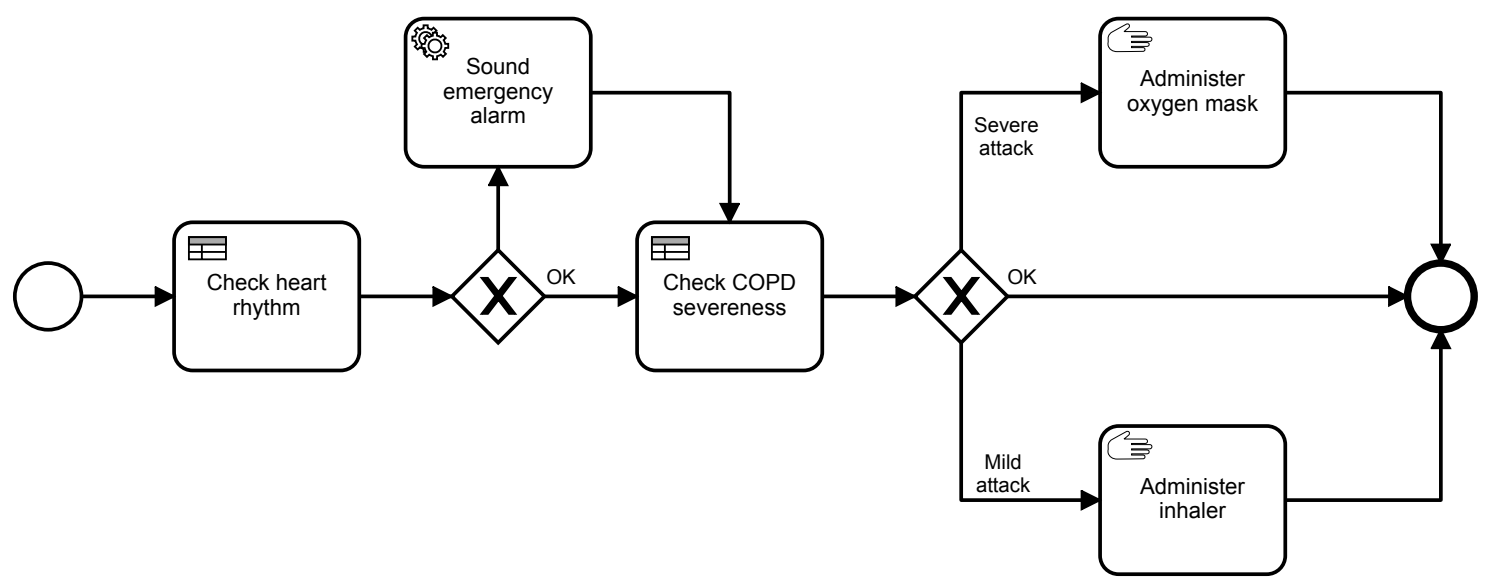

Figure 1: A process model for a COPD monitoring IoT process.

of events that are triggered by an IoT device. If an IoT device registers an action in the physical world, this is reflected in the process by triggering an event. The execution of the event can subsequently trigger a part of the process. In the other direction, the process can trigger the execution of an IoT device if a certain event manifests itself.

3) Resources. Note that the IoT devices can be viewed as resources of an IoT process. According to the BPMN 2.0 standard specification [6], the resource class in the meta model is used to specify resources that can be referenced by activities. These Resources can be human resources, but also any other resource assigned to activities during process execution. Furthermore, the standard defines resources as abstract, i.e. it only defines the resources without specifying which specific resource is allocated to the process element. Actual resource allocation is resolved at runtime. Given that IoT devices can be viewed as resources, the integration of IoT technology and business processes can be approached in a BPMN 2.0 compliant manner. Consider IoT devices as resources which can be invoked from the process model. Since the standard specification considers abstract resources, this can be achieved without administering changes or extensions to the standard BPMN specification. Furthermore, the existing BPMN 2.0 elements with automation properties, such as service tasks and business rule tasks, can be used to execute the IoT process.

4) Data. Notice that the data interchange between the IoT devices on the one hand and the IoT process on the other

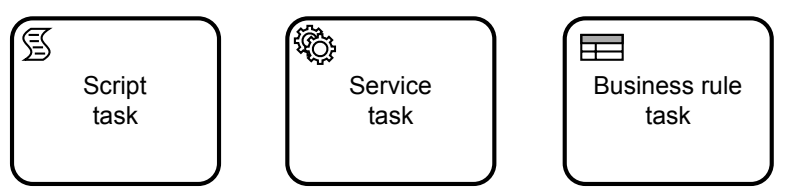

Figure 2: BPMN 2.0 script, service, and business rule tasks. is of paramount importance in a highly interconnected and dynamic setting where the IoT data drives the decisions made in the process. Such a setting was illustrated in the running example in Section III, where the decisions in the process are driven by a data collected from multiple IoT devices. The IoT infrastructure produces plenty of data that can be relevant for the process. This data needs to be interpreted and correctly utilised by the IoT process. Thus, IoT processes are strongly data-intensive as the data perspective of IoT processes revolves around data elements being consumed, produced and exchanged during process execution. This emphasises that a critical resource for an IoT process is the IoT data itself.

\section{Challenges}

Although the BPMN 2.0 standard already offers good support for different aspects of IoT process execution, some challenges still remain. Here, we enumerate a set of research challenges that need to be addressed to guarantee a sound IoT process execution, relying on the BPMN 2.0 standard. For each challenge we follow the same structure: we briefly describe the challenge, provide an illustration based on the running example in Section III] and describe relevant literature that tries to approach the research gap described in the challenge. An overview of the challenges is provided in Table I

\section{A. Resource allocation challenges}

Inherent in IoT processes is the use of IoT devices as resources for task automation and context adaptation. In what follows, we point out challenges for IoT resource awareness in processes.

\section{Challenge 1 (C1): IoT resource-awareness in tasks}

1) Challenge description: In the previous section, we discussed the current support of the BPMN language towards incorporating IoT devices in the business process from a modelling perspective. The challenge is to configure the IoT process in a technology-independent way, yet with a sufficient level of detail to interact with the available resources at execution time. 
2) Example: Take for instance the sound emergency alarm service task of the running example in Figure 11. The service task is linked to the alarm device in the connected reality. The execution of the alarm device corresponds to the execution of the sound emergency alarm service task. Here it is clear that the business process is guiding the use of the IoT technology in the physical world. However, as previously mentioned, the other direction of integrating IoT devices and the business process is a possibility as well. Namely, the IoT devices can be used to digitalise the context in which the business process is executed. This can for instance happen through the check heart rhythm and check COPD severeness business rule tasks in the process model in Figure 1 . The logic hidden behind the business rule tasks is dependent on data collected from the IoT resources.

3) Possible solutions: Researchers have argued that BPMN 2.0 is capable of modelling IoT processes without necessarily extending the existing standard specification. For instance, [13] shows how BPMN elements can be used to model Wireless Sensor Network Applications. The devices and actions performed with the devices are not represented in the model. Thus, no discrimination between regular automated tasks and IoT tasks is present in these models, both when it comes to process modelling as well as process execution. The service tasks simply call upon a service responsible for managing an IoT device. Others, however, have indicated the need to distinguish between regular automated tasks and IoT tasks in the design phase. They propose a visual discrimination between regular automated tasks and IoT tasks, as well as between regular events and IoT events [5]. They link IoT resources to the process by adding a new symbol indicating that an activity or event is linked to an IoT resource, and they also extend the meta model to include the newly proposed types of IoT tasks and events. These newly defined process elements can be made executable through code generation, by extending existing process engines to incorporate the new elements, or by developing a custom process engine. Note that such an approach deviates from the standard BPMN 2.0 support as described in Section IV. Hence, a trade off exists between conforming to the standard specification or extending it for IoT purposes. The former guarantees that the IoT process remains executable according to BPMN 2.0, while the latter explicitly models IoT devices in the process.

Approaches modelling the IoT device within the business process are straightforward as they resort to a one-to-one mapping between process elements and IoT devices. However, a one-to-one mapping between an IoT device and a process element is not necessarily satisfactory. Furthermore, IoT processes can rely on multiple IoT devices, even within the same process element. Therefore, rule-based IoT resource allocation should be investigated, in analogy with works on regular resource allocation and scheduling in business processes [17].

\section{Challenge 2 (C2): IoT resource binding at runtime}

1) Challenge description: In an IoT infrastructure, multiple devices that monitor or execute the same physical reality can be put in place. In such a setting, rather than a straightforward one-to-one mapping of IoT resources and process elements, as described in the previous challenge, a selection of the proper resource at runtime is necessary. This link between the process model and the IoT devices should be established depending on certain criteria, such as device availability, accuracy, malfunctioning, and so on.

2) Example: Take for instance the sound emergency alarm service task of the running example in Figure 1 which is linked to an alarm device in the connected reality. The most relevant alarm device can be selected for execution, e.g. the speakers present on the floor of the hospital where the patient is, or the smart phone of the physician that monitors the patient if the physician is currently not on the same floor.

3) Possible solutions: Optimised resource allocation is considered an important challenge in the merger of IoT technologies and business processes [4]. Among other solutions, the logic on which device to select at runtime can be modelled in a decision model. A standard suitable for such decision modelling is the Decision Model and Notation (DMN) [18] provided by the Object Management Group. This model can be invoked at runtime to dynamically bind an IoT device to the relevant process element. That way, a more efficient IoT resource utilisation can be achieved and process execution can be safeguarded. A similar approach that aims at resolving IoT resource conflicts in business processes is suggested in [19]. Here, the authors define event condition action (ECA) statements indicating when and under which conditions an already allocated resource should be substituted with an equivalent resource.

\section{Challenge $3(\mathrm{C3})$ : IoT resource malfunction event handling}

1) Challenge description: Once IoT resources have been allocated to a process element, be it by a simple one-toone mapping as discussed in $C l$ or by dynamic resource binding at runtime as considered in $C 2$, the IoT resource may still be deficient or inaccessible during the enactment of the relevant process elements. These unexpected events should be captured in the process. In case an allocated IoT device is not responding or failing, the IoT process might not be able to continue its execution. Such failures should be noticed and captured in the process. Resource failure monitoring is crucial for the quality of task execution [4].

2) Example: In the running example of Figure 11, such a safeguard should be foreseen at service task sound emergency alarm. If the alarm device fails during execution, the consequences for the patient being monitored are potentially severe.

3) Possible solutions: In the case described in the example, opting for process termination is not an option. Therefore, a routine should be put in place to select a different alarm device or to convey the message to the physician in some other manner, e.g. by sending a text message or smart notification to the physician's smart phone or by initiating an automated call. In case of multiple alarm devices, by re-invoking the service task responsible for the operation of the failing device, an alternative device can be dynamically bound to the service 
task. A modelling pattern should be foreseen in the process model to capture failures and to call upon another IoT device to perform the execution. Remedying resource malfunction complications can be done by for instance setting up error event handling modelling patterns to assure that processes can continue, terminate, restart, or that they can be partially rolled back to reiterate the failed activities with other available resources. In [13], some error handling patterns for wireless sensor networks (WSN) have been introduced. The patterns rely on events that capture undesirable behaviour. Another interesting line of research are self-healing business processes. Here, the idea is to replace at runtime services that fail during process execution. An example is provided in [20], where the authors define monitoring and recovery actvities to ensure process resilience.

\section{Challenge 4 (C4): IoT data retrieval: push vs. pull}

1) Challenge description: Another important challenge to consider is understanding the context situation in which the process is being executed. This can be done by pushing the data to the process from the IoT devices or by the process pulling the data from the devices on demand. In the former case a sensor performs a data push to the process. However, given that IoT devices often provide a stream of data, the data pushes are likely to happen frequently.

2) Example: In the running example of Figure 11, the data necessary for invoking the business rule activities check heart rhythm and check COPD severeness can be pulled from the IoT sensors described in the running example in Section III. Notice however, that a data push from the IoT devices to the process might be required as well in certain situations. If the heart rhythm sensor notices a steep increase of the heart rhythm which is followed by a quick decrease in a short period of time, this might point towards heart arrhythmia. If the heart rhythm sensor captures such behaviour, the IoT device should push a notification to the process. A data push from the IoT device to the process can for instance be captured in the process through an event, which in turn can trigger activities that update the patient's medical file and that automatically warn a responsible physician.

3) Possible solutions: As the example illustrates, a trade off between pushing IoT data to the process and providing the process with IoT data on demand should be made when setting up the system to guarantee effective process enactment. The data can be captured in the process by, for instance, events that are triggered when the IoT device pushes data to the process. Another possibility is for the process to pull the data captured by the devices on demand. The devices register data and store it into a database or knowledge base, if the most current data update by the devices is considered relevant. In such a way, the data stored in the database or knowledge base is the most recent relevant data. The process can then pull necessary data from the database whenever it is needed in the process. This can for instance be done by using a service task which queries the database and retrieves the necessary data. This data can subsequently be used in the following stages of the process.
Additionally, a combination of push and pull is possible as well, i.e. some IoT devices store data in the database such that the process can pull data on demand. Other IoT devices immediately notify the process by for instance triggering an event in the process that captures the sent data and provides it to the process for further interpretation.

Existing work on the integration of ontologies and task models [21], [22] in IoT environments, as well as the integration of ontologies and Petri Nets [2] can provide inspiration towards solving the data retrieval challenge. Furthermore, relevant works also include those that provide possibilities to retrieve data based on semantic queries over ontologies [23].

\section{B. Communication challenges}

Introducing IoT resources to the business process invokes additional questions regarding the communication between the business process and said resources. We elaborate further below.

\section{Challenge 5 (C5): IoT and human resource communication}

1) Challenge description: Regular non-automated processes tasks are usually performed by human actors. Whenever users finish a task that has been assigned to them, they indicate this by sending a confirmation to the system. This way, the next task in line becomes enabled and the human resource responsible for the enactment of that task can start with the execution. Notice, however, that in an IoT setting task automation is a commonplace pattern. Hence, certain tasks will not be executed by human resources, but rather by the system itself. This contributes to the co-existence of different types of resources in an IoT process [4]. In such a setting, the system needs to notify the process that the automated task has been executed successfully such that subsequent tasks, be they human tasks or automated system tasks, become enabled. If the subsequent tasks are driven by human resources, the system should notify the users about system task completion and it should indicate that the tasks the human resources are responsible for are permitted to be executed. Thus, a message notification system should be put in place to facilitate the interaction between automated IoT tasks and human user-dependent tasks. Furthermore, these message notifications should be organised in such a way that they maximise effectiveness, i.e. that they do not needlessly bother the receivers of the notifications and that they do not produce non-value adding overhead tasks for the human users.

2) Example: In the running example of Figure 1, the sound emergency alarm activity might be executed for a patient and a nurse responsible for the manual activity administer oxygen mask starts a shift after the alarm, or the nurse was not able to hear the alarm at the time. In that case the nurse should be notified to indeed administer the oxygen mask to the patient, but to be careful not to move the patient into a bad position given the abnormal heart rhythm as established in the check heart rhythm decision activity.

1) Possible solutions: In [24] simple message flows in BPMN between IoT tasks and human tasks have been proposed to facilitate co-existent resource communication. A 
more advanced approach can be found in [25], where the authors propose considerate notifications which interact with users in a way that is not disturbing for the user. They provide a possibility to personalise and adjust the level of obtrusiveness of the notifications.

\begin{tabular}{l}
\hline Research challenges in BPMN 2.0 IoT process execution \\
\hline Resource allocation challenges \\
C1: IoT resource-awareness in tasks \\
C2: IoT resource binding at runtime \\
C3: IoT resource malfunction event handling \\
C4: IoT data retrieval: push vs. pull \\
Communication challenges \\
C5: IoT tasks and human resources communication
\end{tabular}

Table I: Research challenges in IoT process execution.

\section{Conclusion And Future Work}

This paper discusses the configuration and execution of IoT-enhanced processes. More specifically, we investigated to which extent the latest BPMN standard supports IoT process execution. To manage this, we consulted existing works on the integration of IoT and processes and we concluded that most research revolves around the modelling of IoT devices in processes. Only a select group of researchers also touch on the subject of configuring and executing the IoT-enhanced process models by either relying on already existing process engines, custom process engines, or by utilising code generation. As most researchers rely on BPMN 2.0 for the modelling effort, we argue that sticking to the core of the standard is beneficial for IoT process execution as well. However, we identified a set of challenges regarding IoT process configuration and execution according to BPMN 2.0. Furthermore, we analysed the literature to identify approaches that address these challenges. However, the approaches presented in literature are either not standardised or not sufficient to tackle the challenges. Therefore, we appeal that these challenges need to be fully remedied to obtain a clear view on IoT process execution.

The identified challenges should be addressed in the future with the aim to solidify the integration of IoT and business processes for execution. Particular attention will be given to alleviate the burdens specified in the research challenges in this paper. More specifically, we will focus on IoT resourceawareness in process models, resource malfunctioning, as well as on dynamic IoT resource binding at runtime. Finally, we will study change patterns for IoT process evolution at runtime.

\section{REFERENCES}

[1] K. Ashton et al., "That internet of things thing," RFID journal, vol. 22, no. 7, pp. 97-114, 2009.

[2] E. Serral, J. De Smedt, M. Snoeck, and J. Vanthienen, "Context-adaptive petri nets: Supporting adaptation for the execution context," Expert Systems with Applications, vol. 42, no. 23, pp. 9307-9317, 2015.

[3] M. Hassanalieragh, A. Page, T. Soyata, G. Sharma, M. Aktas, G. Mateos, B. Kantarci, and S. Andreescu, "Health monitoring and management using internet-of-things (iot) sensing with cloud-based processing: Opportunities and challenges," in 2015 IEEE international conference on services computing (SCC). IEEE, 2015, pp. 285-292.
[4] C. Janiesch, A. Koschmider, M. Mecella, B. Weber, A. Burattin, C. Di Ciccio, A. Gal, U. Kannengiesser, F. Mannhardt, J. Mendling et al., "The internet-of-things meets business process management: mutual benefits and challenges," arXiv preprint arXiv:1709.03628, 2017.

[5] A. Yousfi, C. Bauer, R. Saidi, and A. K. Dey, "ubpmn: A bpmn extension for modeling ubiquitous business processes," Information and Software Technology, vol. 74, pp. 55-68, 2016.

[6] OMG, "Business process model and notation (BPMN) 2.0," 2011.

[7] G. Hermosillo, L. Seinturier, and L. Duchien, "Creating context-adaptive business processes," in International Conference on Service-Oriented Computing. Springer, 2010, pp. 228-242.

[8] J. Hu, G. Aghakhani, F. Hasić, and E. Serral, "An evaluation framework for design-time context-adaptation of process modelling languages," in IFIP Working Conference on The Practice of Enterprise Modeling. Springer, 2017, pp. 112-125.

[9] OMG, "Xml metadata interchange (XMI) 2.5.1," 2015.

[10] Camunda, "Process engine," https://docs.camunda.org/manual/7.8/userguide/process-engine/, 2018, accessed: 2018-11-16.

[11] S. Meyer, A. Ruppen, and C. Magerkurth, "Internet of things-aware process modeling: integrating iot devices as business process resources," in International conference on advanced information systems engineering. Springer, 2013, pp. 84-98.

[12] N. Brouns, S. Tata, H. Ludwig, E. S. Asensio, and P. Grefen, "Modeling iot-aware business processes-a state of the art report," arXiv preprint arXiv:1811.00652, 2018.

[13] A. Caracaş and T. Kramp, "On the expressiveness of bpmn for modeling wireless sensor networks applications," in Business Process Model and Notation, R. Dijkman, J. Hofstetter, and J. Koehler, Eds. Berlin, Heidelberg: Springer Berlin Heidelberg, 2011, pp. 16-30.

[14] D. Domingos and F. Martins, "Using bpmn to model internet of things behavior within business process," IJISPM-INTERNATIONAL JOURNAL OF INFORMATION SYSTEMS AND PROJECT MANAGEMENT, vol. 5, no. 4, pp. 39-51, 2017.

[15] S. Appel, P. Kleber, S. Frischbier, T. Freudenreich, and A. Buchmann, "Modeling and execution of event stream processing in business processes," Information Systems, vol. 46, pp. 140-156, 2014.

[16] T. Peng, C. Chi, A. Chiasera, G. Armellin, M. Ronchetti, C. Matteotti, C. Parra, A. O. Kashytsa, and A. Varalta, "Business process assignment and execution in mobile environments," in 2014 International Conference on Collaboration Technologies and Systems (CTS), May 2014, pp. 267-274.

[17] P. Wohed, W. M. van der Aalst, M. Dumas, A. H. ter Hofstede, and N. Russell, "On the suitability of bpmn for business process modelling," in International conference on business process management. Springer, 2006, pp. 161-176.

[18] OMG, "Decision Model and Notation (DMN) 1.2," 2018.

[19] K. Suri, W. Gaaloul, A. Cuccuru, and S. Gerard, "Semantic framework for internet of things-aware business process development," in Enabling Technologies: Infrastructure for Collaborative Enterprises (WETICE), 2017 IEEE 26th International Conference on. IEEE, 2017, pp. 214219.

[20] L. Baresi, S. Guinea, and L. Pasquale, "Self-healing bpel processes with dynamo and the jboss rule engine," in International workshop on Engineering of software services for pervasive environments: in conjunction with the 6th ESEC/FSE joint meeting. ACM, 2007, pp. $11-20$.

[21] E. Serral, P. Valderas, and V. Pelechano, "Context-adaptive coordination of pervasive services by interpreting models during runtime," The Computer Journal, vol. 56, no. 1, pp. 87-114, 2012.

[22] — "Addressing the evolution of automated user behaviour patterns by runtime model interpretation," Software \& Systems Modeling, vol. 14, no. 4, pp. 1387-1420, 2015.

[23] D. Calvanese, B. Cogrel, S. Komla-Ebri, R. Kontchakov, D. Lanti, M. Rezk, M. Rodriguez-Muro, and G. Xiao, "Ontop: Answering sparql queries over relational databases," Semantic Web, vol. 8, no. 3, pp. 471487, 2017.

[24] R. Pryss, M. Reichert, A. Bachmeier, and J. Albach, "Bpm to go: Supporting business processes in a mobile and sensing world," in BPM Everywhere, 2015, pp. 167-182. [Online]. Available: http://dbis.eprints.uni-ulm.de/1153/

[25] E. Serral, M. Gil, P. Valderas, and V. Pelechano, "Automating unobtrusive personalized services in ambient media environments," Multimedia tools and applications, vol. 71, no. 1, pp. 159-178, 2014. 